\title{
Dirty $\mathrm{H}_{2}$ Molecular Clusters as the DIB Sources: Spectroscopic and Physical Properties
}

\author{
L. S. Bernstein ${ }^{1}$, F. O. Clark ${ }^{1}$ and D. K. Lynch ${ }^{2}$ \\ ${ }^{1}$ Spectral Sciences Inc., 4 Fourth Avenue, Burlington, MA 01803, USA \\ email: larry@spectral.com \\ ${ }^{2}$ Thule Scientific, P.O. Box 953, Topanga CA 90290, USA \\ email: dave@thulescientific.com
}

\begin{abstract}
We propose that the diffuse interstellar bands (DIBs) arise from absorption lines of electronic transitions in molecular clusters primarily composed of a single molecule, atom, or ion ( "seed"), embedded in a single-layer shell of $\mathrm{H}_{2}$ molecules (Bernstein et al. 2013). Less abundant variants of the cluster, including two seed molecules and/or a two-layer shell of $\mathrm{H}_{2}$ molecules may also occur. The lines are broadened, blended, and wavelength-shifted by interactions between the seed and surrounding $\mathrm{H}_{2}$ shell. We refer to these clusters as CHCs (Contaminated $\mathrm{H}_{2}$ Clusters). CHC spectroscopy matches the diversity of observed DIB spectral profiles, and provides good fits to several DIB profiles based on a rotational temperature of $10 \mathrm{~K}$. CHCs arise from $\sim \mathrm{cm}$-sized, dirty $\mathrm{H}_{2}$ ice balls, called CHIMPs (Contaminated $\mathrm{H}_{2}$ Ice Macro-Particles), formed in cold, dense, Giant Molecular Clouds (GMCs), and later released into the interstellar medium (ISM) upon GMC disruption. Attractive interactions, arising from Van der Waals and ion-induced dipole potentials, between the seeds and $\mathrm{H}_{2}$ molecules enable CHIMPs to attain cm-sized dimensions. When an ultraviolet (UV) photon is absorbed in the outer layer of a CHIMP, it heats the icy matrix and expels CHCs into the ISM. While CHCs are quickly destroyed by absorbing UV photons, they are replenished by the slowly eroding CHIMPs. Since CHCs require UV photons for their release, they are most abundant at, but not limited to, the edges of UV-opaque molecular clouds, consistent with the observed, preferred location of DIBs. An inherent property of CHCs, which can be characterized as nanometer size, spinning, dipolar dust grains, is that they emit in the radio-frequency region. Thus, CHCs offer a natural explanation to the anomalous microwave emission (AME) feature in the $\sim 10-100 \mathrm{GHz}$ spectral region.
\end{abstract}

Keywords. ISM: lines and bands, molecular data, infrared: ISM

\section{Modeling DIBs with CHC Spectroscopy}

We focus on the properties of CHCs with a single seed and a single layer of $\mathrm{H}_{2}$ molecules, since, they are expected to be the most abundant type of CHC (see Fig. 1). A CHC's size depends on the size of its seed and the number of $\mathrm{H}_{2}$ layers bound to the seed by the seed- $\mathrm{H}_{2}$ potential. Small molecular seeds, such as $\mathrm{CO}$ and $\mathrm{CH}_{4}$, whose interaction potential with $\mathrm{H}_{2}$ is weakly dependent on molecular orientation, will rotate freely within the surrounding $\mathrm{H}_{2}$ shell. Larger, non-spherical seeds, such as $\mathrm{CO}_{2}$ and $\mathrm{C}_{3} \mathrm{H}_{2}$, will be locked into position by the $\mathrm{H}_{2}$ layer, and, seed rotation will be rigidly tied to that of the whole cluster. Most seeds will form "locked" CHCs. Geometrically, a CHC's $\mathrm{H}_{2}$ layer(s) will approximately conform to the shape of its seed, resulting in a spherical, symmetric, or asymmetric top cluster. Thus, CHC spectral profiles will reflect the spectroscopy for these classes of molecular symmetry. The idea that some of the structured DIB spectral profiles can be modeled well in terms of a large linear or symmetric top molecule has been demonstrated by several groups (Danks \& Lambert 1976; Kerr et al. 1996; Walker et al. 


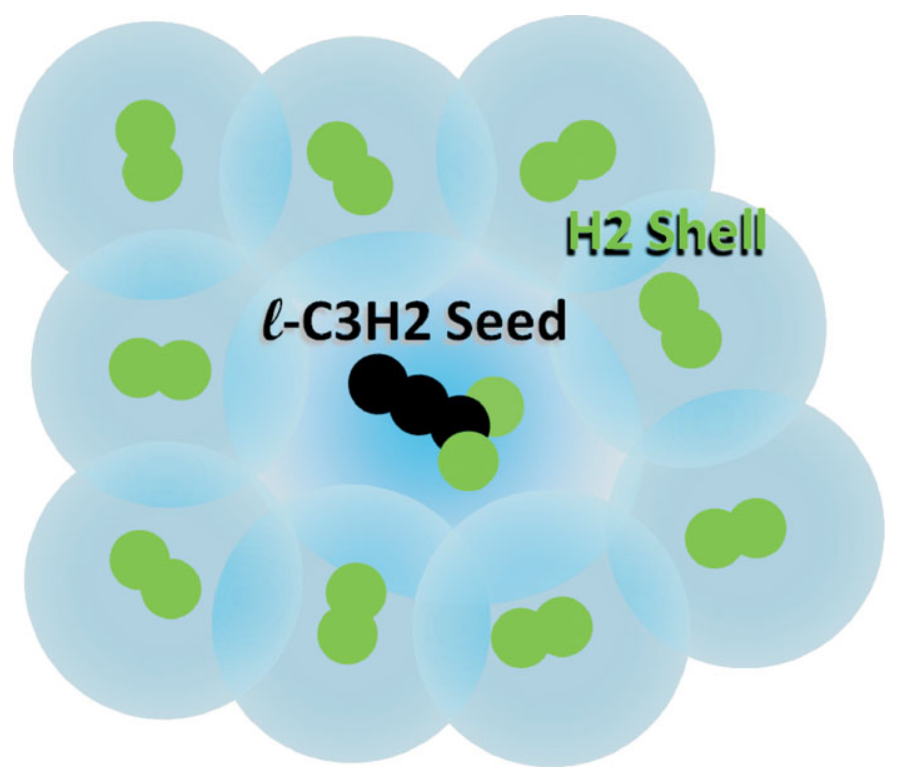

Figure 1. Example CHC.

2001). Here we explore this idea in the context of the physical and spectral parameter constraints associated with $\mathrm{CHC}$ spectroscopy.

DIB wavelengths do not match those of any isolated molecule or atom, because, for CHCs, the transition wavelengths are shifted by interactions between the seed and the $\mathrm{H}_{2}$ molecules. Since a CHC can be characterized as a seed embedded in a frozen molecular matrix. The shifts in the vibration and vibration-electronic transition wavelengths have been measured in Ne and Ar matrices for a wide variety of organic molecules (Jacox 1998; Maier et al. 2004; Nagarajan \& Maier 2010). They fall in the range of $\sim-150$ to $+100 \mathrm{~cm}^{-1}(\sim+58$ to $-39 \AA$ at $6000 \AA)$. Molecular beam experiments by Kuma et al. (2011) demonstrated shifts for tetracene $\left(\mathrm{C}_{18} \mathrm{H}_{12}\right)$ embedded in large $\mathrm{Ne}$, Ar, and $\mathrm{H}_{2}$ clusters of $\sim-30,-200$, and $-450 \mathrm{~cm}^{-1}$, respectively. This implies that shifts for CHC seeds due to $\mathrm{H}_{2}$ may generally be much larger than for $\mathrm{Ne}$ and $\mathrm{Ar}$, and that red shifts of up to $\sim-450 \mathrm{~cm}^{-1}(\sim+174 \AA)$, and possibly larger, are plausible.

For spectral modeling, we assume that symmetric tops are appropriate representations for most CHCs. The spectroscopic parameters for symmetric top CHCs include two rotational constants, $\mathrm{B}$ and $\mathrm{C}$, for the lower and upper states of a transition, the centrifugal distortion constants, $\mathrm{D}_{J}, \mathrm{D}_{K}, \mathrm{D}_{J K}$, the interstellar Doppler velocity spread and Lorentz collision/lifetime broadening parameters, $\gamma_{D}$ and $\gamma_{L}$, the symmetries of the states of the transition (e.g., A, E,...), and the electronic spin and orbital angular momentum of the states. A subset of these parameters suffices to demonstrate the utility of symmetric top modeling of DIBs, i.e., B, C, $\gamma_{D}$ and $\gamma_{L}$.

The CHC symmetric top model was used to fit several well-characterized DIB spectra (Kerr et al. 1996; Walker et al. 2001; Snow et al. 2002; Galazutdinov et al. 2002; Galazutdinov et al. 2008). The results, shown in Figure 2, are based on adjustment, by hand, of the subset of spectroscopic parameters discussed above, and a fixed temperature of $10 \mathrm{~K}$. The fit parameters are listed in Table 1 of Bernstein et al. (2013). The $10 \mathrm{~K}$ fit temperature was selected because it falls in the mid-range of expected CHC temperatures, 3-15 K, needed for CHC stability. These four DIBs are fit as prolate tops, consistent with a linear or hydrocarbon chain seed. 
L. S. Bernstein, F. O. Clark \& D. K. Lynch

CHC Spectroscopy Consistent with DIB Profiles
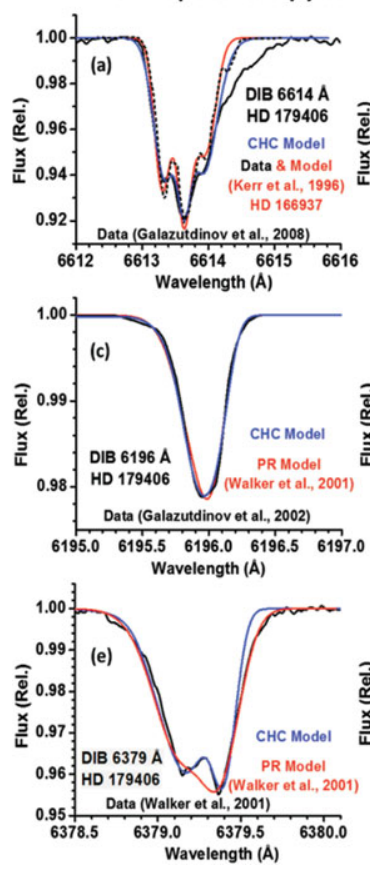
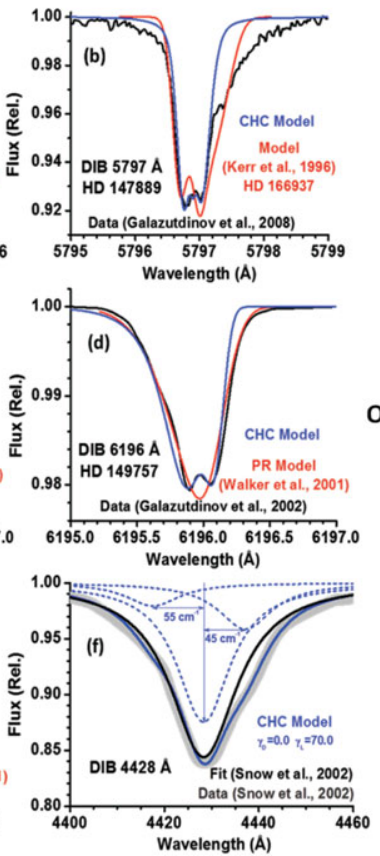

CHCs New Source of AME

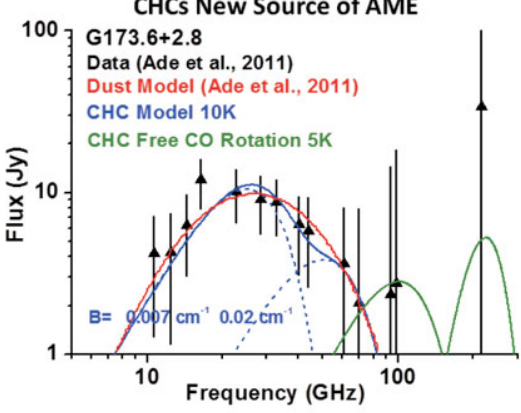

One Seed, Many DIBS Carbon Chain Anions Favored

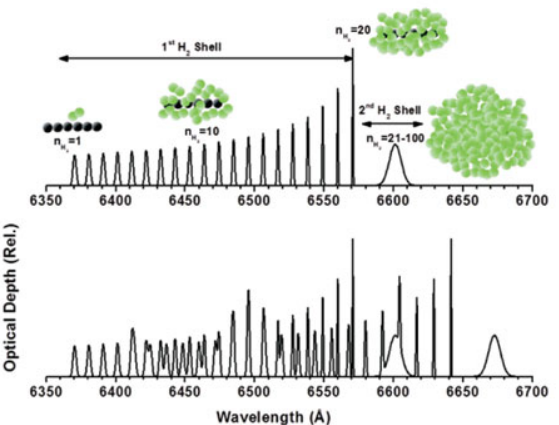

Figure 2. Comparison of CHC model fits to observed DIBs and other models (left), and to an observed anomalous microwave emission (AME) spectrum and spinning dust model fit (upper right) (Draine \& Lazarian 1998; Ade et al. 2011). Also shown is a simulated DIB absorption spectra for a single-seeded $\mathrm{CHC}$ as a function of the number of $\mathrm{H}_{2}$ molecules in its surrounding $\mathrm{H}_{2}$ shells for a single rovibronic seed transition (bottom right, top) and two, overlapping rovibronic transitions (bottom right, bottom).

\section{Acknowledgements}

L. B. and F. C. appreciate Spectral Sciences, Inc. funding.

\section{References}

Ade, P. A. R., et al. Planck Collaboration 2011, A\& A, 536, A20

Bernstein, L. S, Clark, F. O., \& Lynch, D. K. 2013, ApJ, 768, 84

Danks, A. C. \& Lambert, D. L. 1976, MNRAS, 174, 571

Draine, B. T. \& Lazarian, A. 1998, ApJL, 494, L19

Galazutdinov, G. A., LoCurto, G., \& Krelowski, J. 2008, ApJ, 682, 1076

Galazutdinov, G., Moutou, C., Musaev, F., \& Krelowski, J. 2002, A\&\&A,384, 215

Jacox, M. E. 1998, J. Phys. Chem. Ref. Data, 27, 115

Kerr, T. H., Hibbins, R. E., Miles, J. R., et al. 1996, MNRAS, 283, L105

Kuma, S., Nakahara, H., Tsubouchi, M., et al. 2011, JPhCh, 115, 7392

Maier, J. P., Walker, G. A. H., \& Bohlender, D. A. 2004, ApJ, 602, 286

Nagarajan, R. \& Maier, J. P. 2010, IRPC, 29, 521

Snow, T. P., Zukowski, D., \& Massey, P. 2002, ApJ, 578, 877

Walker, G. A. H.,Webster, A. S., Bohlender, D. A., \& Krelowski, J. 2001, ApJ, 561, 272 